\title{
Nanoindentation Test Conducted on Single Scan Tracks for the Development of New Multi-Principal Element Alloys
}

Giulio Cattano (Department of Applied Science and Technology, Politecnico di Torino, Corso Duca degli Abruzzi 24, 10129 Torino, Italy) giulio.cattano@polito.it; giulio.cattano@iit.it; Massimo Lorusso (Centre for Sustainable Future Technologies CSFT@PoliTo, Istituto Italiano di Tecnologia, Corso Trento 21, 10129, Torino, Italy) massimo.Iorusso@iit.it; Flaviana Calignano (Department of Management and Production Engineering, Corso Duca degli Abruzzi 24, 10129 Torino, Italy) flaviana.calignano@polito.it; Marco Actis Grande (Department of Applied Science and Technology, Politecnico di Torino, Corso Duca degli Abruzzi 24, 10129 Torino, Italy) marco.actis@polito.it; Diego Manfredi (Centre for Sustainable Future Technologies CSFT@PoliTo, Istituto Italiano di Tecnologia, Corso Trento 21, 10129, Torino, Italy) diego.manfredi@iit.it; Mariangela Lombardi (Department of Applied Science and Technology, Politecnico di Torino, Corso Duca degli Abruzzi 24, 10129 Torino, Italy) mariangela.Iombardi@polito.it; Paolo Fino (Department of Applied Science and Technology, Politecnico di Torino, Corso Duca degli Abruzzi 24, 10129 Torino, Italy) paolo.fino@polito.it

\section{Abstract}

Urgent environmental challenges and emerging additive manufacturing (AM) technologies push research towards more performant and new materials. In the field of metallurgy, high entropy alloys (HEAs) have recently represented a topic of intense research because of their promising properties, such as high temperature strength and stability. Moreover, this class of multi-principal element alloys (MPEAs) have opened up researcher community to unexplored compositional spaces, making prosper literature of high-throughput methodologies and tools for rapidly screening large number of alloys. However, none of the methods has been aimed to design new MPEAs for AM process known as selective laser melting (SLM) so far. Here we conducted nanoindentation testing on single scan tracks of elemental powder blends and pre-alloyed powders after ball milling of AITiCuNb and AITiVNb. Results show that nanoindentation can represent an effective technique to gain information about phase evolution during laser scanning, contributing to accelerate the development of new MPEAs.

\section{Introduction}

Additive manufacturing (AM) laser powder bed fusion (LPBF) process commonly known as selective laser melting (SLM), has been addressing increasing interests in processing new materials. In SLM a computer-controlled laser beam locally melts single layers of metal powders, which rapidly solidify creating unique microstructures. However, the possibility of a significant material development using traditional alloy design is still limited.

In a high entropy alloy (HEA), the base alloy has significant atomic fractions of several elements. Differently to conventional alloys - which consist of one principal element and few quantities of other elements - HEAs are nominally formed by five or more elements in equal or near-equal atomic concentration [1]. In many cases, because of the chemical heterogeneity, HEAs may exhibit complex microstructures with brittle intermetallics or other complex ordered phases [2]. However, the selection of starting elements, together with the high entropy of mixing, may lead to the stabilization of simple single phase microstructures, either face-centered cubic (FCC) or body-centered cubic (BCC) $[1,3]$ characterized by a broad range of interesting properties, such as high thermal stability [4] and strength [5]. It is worth noting that in the last years also systems with $<5$ principal elements in equal-atomic concentration, were found to exhibit simple solid solution phases [6,7]. For this reason, the more general term of 'multi-principal element alloys' (MPEAs) has emerged [8]. Regardless the nomenclatures, due to the vast number of compositions that can be tailored using a multi-principal element design, it was necessary to adopt high-throughput methodologies for rapidly screening a large number of alloy systems. These methods manly include (1) empirical rules to predict the formation of solid solutions $[2,10,11]$ or semiempirical computational Calphad method to assess number and type of phases in certain conditions [8,9] and (2) production of thin films [12] or bulk samples by casting [13] for high-throughput evaluation of material properties. Empirical rules represent a relatively fast and ready-to-use method for alloy screening and they generally use three parameters, the entropy of mixing $\left(\Delta \mathrm{S}_{\text {mix }}\right)$, the enthalpy of mixing $\left(\Delta \mathrm{H}_{\text {mix }}\right)$ and the atomic size parameter $(\delta)$ or a combination of them $[2,10,11]$.

Miracle et al. [14] suggested a theoretical strategy to develop new MPE structural alloys by combining high-throughput Calphad computations as screening tool and high-throughput experiments for 
properties evaluation, i.e EDS mapping and nanoindentation on thin films. As reported in literature, these are well-established screening techniques [12, 15]. Recently, Maier-Kiener et al. [16] has successfully employed nanoindentation testing as a powerful screening tool to detect phase decomposition at high temperature on the equiatomic CoCrFeMnNi HEA produced by casting. Finally, Haase et al. [17] introduced a high-throughput computation and experimental methodology for rapid alloy screening and design, consisting on the production of bulk samples by AM laser metal deposition (LMD) technique starting from elemental powder blends and validated the method on the CoCrFeMnNi Cantor alloy. However, to the best of our knowledge, none of the strategies suggested by now is directly aimed to design new HEAs/MPEAs by SLM.

In the present study, we conducted nanoindentation testing on cross sections of single laser scan tracks (SLSTs) of two MPEAs, AITiCuNb and AITiVNb, obtained from elemental powder mixtures and on AITiCuNb powders obtained by high-energy ball milling. In SLST, a thin layer of powders is laser scanned according to one directional line at fixed laser power and scanning speed. In this way, it could provide significant information on the interaction between laser and powder, crucial in designing new alloys for SLM. Nanoindentation testing is a promising tool for gaining information on phase evolution during SLM and on the mechanical response of the selected systems, contributing to accelerate the development of new MPEAs for SLM.

\section{Materials and method}

The AITiCuNb and AITiVNb equal-atomic mixtures were prepared starting from elemental powders. From now on, they will be called $\mathrm{AlTiCuNb}$ mix and AITiVNbmix, respectively. Sizes, manufacturers and thermo-physical properties of elemental powders are listed in Table 1 and Table 2, respectively. The present alloy systems were selected as they reveal a strong possibility to form a BCC solid solution, according to the empirical solid solution prediction rules proposed by Yang et al. [18] and Guo et al. [19]. Table 3 shows the values for the predictive parameters. They were calculated using the following expressions:

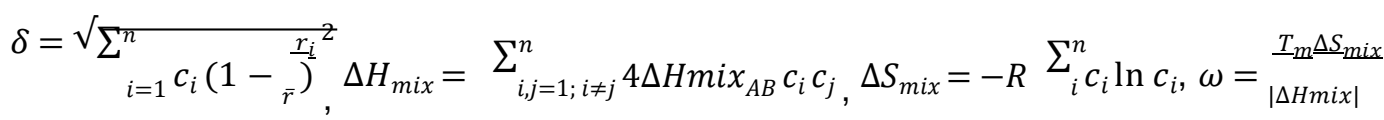

where $r_{i}$ is the atomic radius of the $\mathrm{i}^{\text {th }}$ element, $\bar{r}$ is the mean atomic radius, $\mathrm{C}_{\mathrm{i}}$ and $\mathrm{c}_{\mathrm{j}}$ are the atomic concentration of the $\mathrm{i}^{\text {th }}$ and $\mathrm{j}^{\text {th }}$ component, $\mathrm{R}$ is the universal gas constant and $\mathrm{T}_{\mathrm{m}}$ is the mean melting temperature.

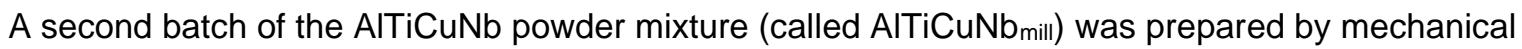
alloying and milled for 20h with hardened steel balls in a high energy Planetary Ball Mill Pulverisette 7 by Fritsch, at $450 \mathrm{rpm}$ in Ar atmosphere and ball-to-powder weight ratio (BPR) 6:1. Phase analysis was performed using a X-Pert Philips diffractometer with a Cu Ka radiation in a Bragg Brentano configuration in a $2 \theta$ range between 20 and 100 degrees (operated at $40 \mathrm{KV}$ and $30 \mathrm{~mA}$ with a $0.02^{\circ}$ step size and 1 sec per step).

Table 1: Composition of metal powders used and their suppliers.

\begin{tabular}{|c|c|c|c|}
\hline Element & Purity (\%) & Particle size ( $\boldsymbol{\mu m})$ & Supplier \\
\hline $\mathrm{Al}$ & 99.5 & $<45$ & Alpha Aesar \\
\hline $\mathrm{Ti}$ & 99.2 & $<45$ & TLS technik \\
\hline $\mathrm{Cu}$ & 99.9 & $5-25$ & Sandvik \\
\hline $\mathrm{Nb}$ & 99.8 & $1-5$ & ABCR \\
\hline $\mathrm{V}$ & 99.9 & $<45$ & Nmd \\
\hline
\end{tabular}

Table 2: Thermo-physical properties of elements.

\begin{tabular}{|l|c|c|c|c|c|c|}
\hline & & $\mathbf{A l}$ & $\mathbf{T i}$ & $\mathbf{C u}$ & $\mathbf{V}$ & $\mathbf{N b}$ \\
\hline $\mathbf{r}(\mathbf{p m})$ & & 143.17 & 146.15 & 127.8 & 131.6 & 142.9 \\
\hline $\left.\mathbf{T}_{\mathbf{m}}{ }^{\circ} \mathbf{C}\right)$ & & 660 & 1668 & 1085 & 1910 & 2477 \\
\hline $\mathbf{V E C}$ & & 3 & 4 & 11 & 5 & 5 \\
\hline$\Delta \mathbf{H}_{\text {A-B }}\left(\mathrm{kJ} \mathrm{mol}^{-1}\right)$ & $\mathbf{T i}$ & -30 & & & & \\
\hline & $\mathbf{C u}$ & -1 & -9 & & & \\
\hline & $\mathbf{V}$ & -16 & -2 & & & \\
\hline & $\mathbf{N b}$ & -18 & 2 & 3 & -1 & \\
\hline
\end{tabular}


Table 3: Theoretical density and solid solution prediction parameters for AITiCuNb and AITiVNb. $\delta$ is the atomic size parameter and $\omega$ is the solid solution formability parameter. $\delta \leq 6.6 \%$ and $\omega \geq 1.1$ are the conditions to have the formation of a solid solution [18].

\begin{tabular}{|c|c|c|c|c|c|c|}
\hline Alloy & $\boldsymbol{\rho}_{\text {th }}\left[\mathbf{g} / \mathbf{c m}^{-3}\right]$ & VEC & $\boldsymbol{\delta}[\%]$ & $\Delta \mathbf{H}_{\text {mix }}\left[\mathbf{k J ~ m o l}^{-1}\right]$ & $\mathbf{\Delta S}_{\text {mix }}\left[\mathbf{J} / \mathbf{K ~ m o l}^{-1}\right]$ & $\boldsymbol{\omega}$ \\
\hline AlTiCuNb & 6 & 5.75 & 5.1 & -13.25 & 11.53 & 1.52 \\
\hline AlTiVNb & 5.5 & 4.25 & 3.9 & -16.25 & 11.53 & 1.38 \\
\hline
\end{tabular}

SLSTs of AlTiCuNbmix, AITiVNbmix and AITiCuNbmill powders were produced by LPBF using an EOS M270 Dual Mode system equipped with a $200 \mathrm{~W}$ Yb fiber-laser beam $(\lambda=1064 \mathrm{~nm})$ with a spot size of $100 \mu \mathrm{m}$, working in a protective Ar atmosphere. A single layer of powders of $50 \mu \mathrm{m}$ thickness was deposited on three different AlSi10Mg substrate disks mounted on the building platform and they were all scanned by the laser beam at the constant power of $195 \mathrm{~W}$ and at four different scanning velocities $(300,400,500,600 \mathrm{~mm} / \mathrm{s})$. Due to the large differences in melting temperatures among single elements, in order to be sure to melt all the elemental constituents, each track was laser scanned three times, consecutively. Figure 1 shows the experimental equipment during laser irradiation.

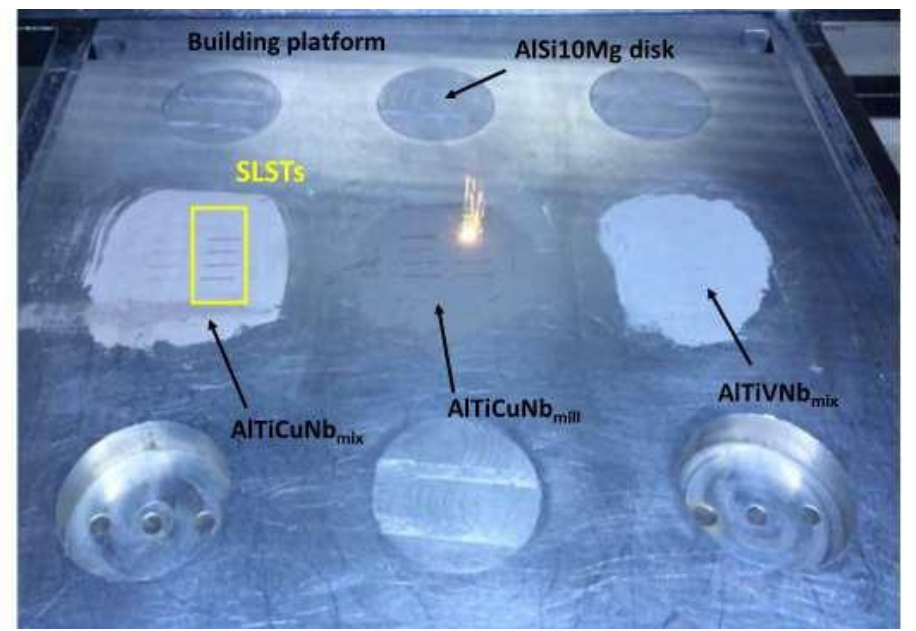

Figure 1: Experimental equipment used to produce SLSTs of AITiCuNb ${ }_{\text {mix }}, \mathrm{AlTiCuNb}_{\text {mill }}$ and $\mathrm{AITiVNb_{ \text {mix } }}$, showing a layer of powder $(50 \mu \mathrm{m})$ during laser irradiation, the AISi10Mg disks mounted on the building platform and the SLSTs obtained after laser scanning (yellow rectangular).

Shapes and morphologies of mixed and milled powders were observed by means of Field Emission Scanning Electron Micrograph (FESEM-FEG) Zeiss SupraTM 40. Cross sections of SLSTs were inspected by means of optical microscope (OM) Leica DMI 5000 M and FESEM-FEG Zeiss SupraTM 40, equipped with an energy dispersive X-ray spectrometer (EDS) for chemical composition.

In order to assess phase evolution of AITiCuNbmix, AITiVNbmix and AITiCuNbmill during SLM and the overall strengthening effect resulting from the multi-principal element design approach, nanoindentation experiments were performed on end-polished cross sections of SLSTs. Nanoindentation testing was carried out using a TI950 nanoindenter (Hysitron) equipped with a diamond Berkovich tip. A controlled load was applied to the specimens and then removed, producing traditional force versus displacement curves. The analysis of the curves, then, provides information regarding the mechanical properties of the specimen. The load was increased at $125 \mu \mathrm{N} / \mathrm{s}$ up a maximum load of $2.5 \mathrm{mN}$ and then decreased at the same rate. Load was applied for $5 \mathrm{sec}$. A grid of $16 \times 13$ indentations, distant $20 \mu \mathrm{m}$ from each other, was realized. For our experiment, the punctual hardness values, expressed in GPa, were plotted using bubble maps.

\section{Results and discussion}

Figure 2 illustrates morphologies of elemental powders of the equal-atomic mixtures $\mathrm{AlTiCuNb} \mathrm{Cm}_{\text {mix }}$ and AITiVNbmix (Figure $2 \mathrm{a}$ and Figure $2 \mathrm{~b}$, respectively). Copper and titanium particles have spherical morphology, aluminium particles have elongated shape and both vanadium and niobium particles exhibit a characteristic plate-like sharp shape. After $20 \mathrm{~h}$ of milling, AlTiCuNbmill powder particles (Figure 2c) show different morphology compared to the elemental powder mixture (Figure 2a). Particles are more aggregated but also more uniform in size as consequence of the repeated welding and fracturing experienced during ball milling. In Figure $2 \mathrm{~d}$, the XRD pattern conducted on the AITiCuNbmill powders shows the formation of a BCC phase, in agreement with the prediction model [18]. Peaks exhibit broadening and weakening as consequence of microstructural refinement during ball milling. 

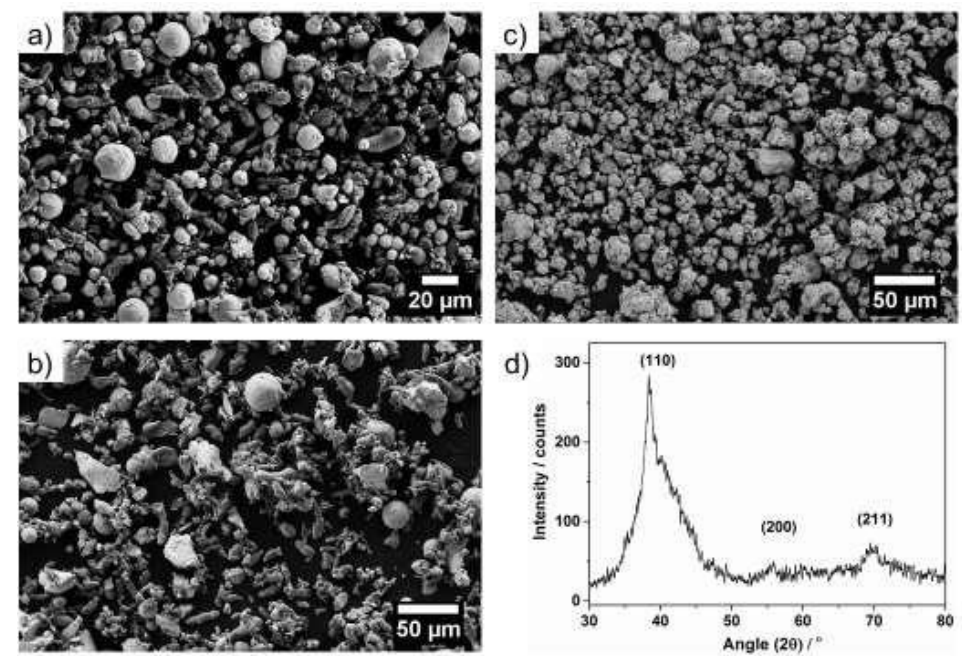

Figure 2: FESEM pictures of a) AITiCuNb ${ }_{\text {mix }}$, b) AITiVNb mix $_{\text {mix }}$ equal-atomic elemental powder mixtures and c) AITiCuNb $\mathrm{Aill}_{\text {m }}$

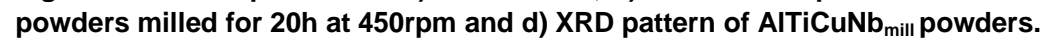

As stated before, consolidation of powders and assessment of the interactions between laser and powders, both in the mixed and milled conditions, were studied by means of SLSTs. Figure 3 shows the cross sections of all SLSTs made. At any conditions, they are characterized by a dome-shaped head and a remelted zone within the support disk. Visual inspection of cross sections reveals that the head is always less developed than the remelting zone and that its height is independent of scanning speed. The triple laser scanning, indeed, may have contributed in flattening the head and producing a deeper remelting zone. However, AITiVNbmix powder seems to interact with laser differently from AITiCuNbmix and AITiCuNbmill, showing a higher head. Furthermore, for any powder type, remelting depth seems to decrease with scanning speeds, especially in AITiCuNbmill. Therefore, in sight of these observations, it can be concluded that both the composition of the MPEAs (AITiCuNb and AITiVNb) and the processing of powders (mixed or milled) affect the morphology of the SLST.

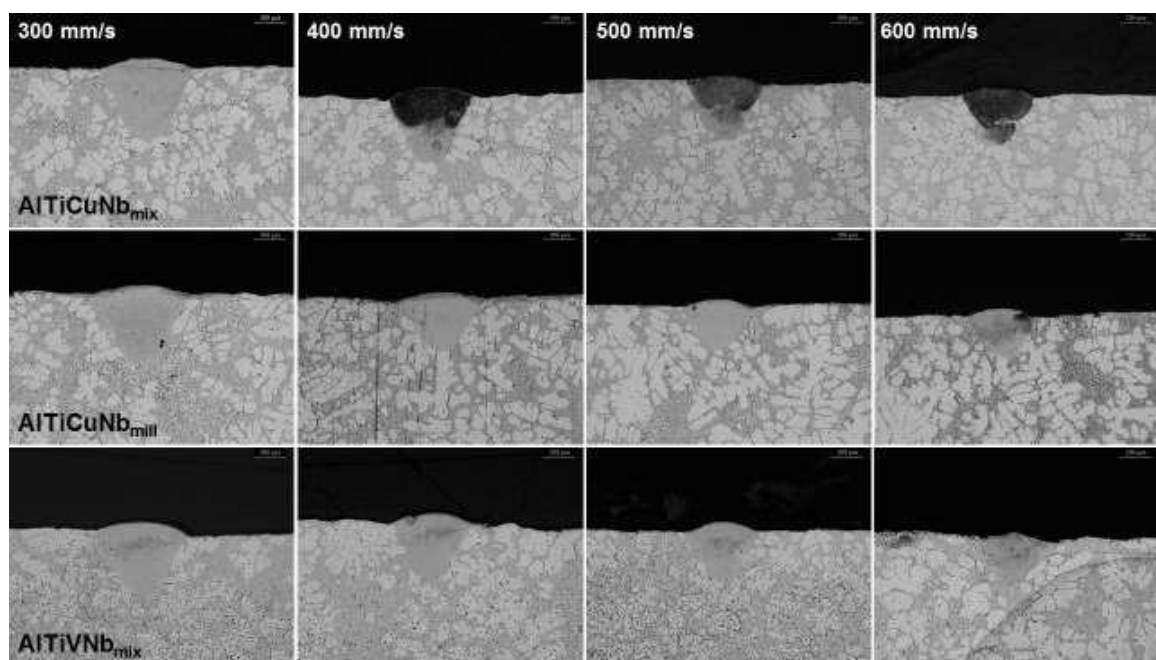

Figure 3: Cross sections of SLSTs of AITiCuNb mix $_{\text {(first row), AITiCuNb }}$ (second row) and AITiVNb mix $_{\text {(third row) at }}$ different scanning speeds $(300,400,500$ and $600 \mathrm{~mm} / \mathrm{s})$. Laser power is constant at $195 \mathrm{~W}$.

Figures $4-5$ and 6 report nanoindentations, OM micrographs, FESEM magnification and EDX quantitative analyses on SLSTs of AITiCuNbmix, AITiCuNbmill and AITiVNbmix respectively, all processed at $500 \mathrm{~mm} / \mathrm{s}$ and $195 \mathrm{~W}$. For any powders, nano hardness bubble maps show a significant strengthening as consequence of the MPEAs compared to the AlSi10Mg support disk (average nano hardness is 1.3 $\mathrm{GPa}$ ). AlTiCuNbmill (Figure 5a) presents the highest hardness homogeneity, likely due to the good dispersion and alloying of elements after the milling process but it is characterized also by the lowest average hardness $(2.7 \mathrm{GPa})$. As one might expect as consequence of the milling process, EDX mapping reveals distribution of $\mathrm{Ti}, \mathrm{Cu}$ and $\mathrm{Nb}$ that is close to the equal-atomic one (Figure $5 \mathrm{c}$ ). Differently, 
AlTiCuNbmix shows a strong Cu enrichment (Figure 4c) and an average hardness of $3.2 \mathrm{GPa}$. For both systems, the overall strengthening is still low, suggesting that AITiCuNb might not be such an interesting system to further develop. On the contrary, AITiVNbmix (Figure 6a), whilst it has a sharp hardness discontinuity near the central region of the remelting zone likely due to element segregations within the melt pool, reveals good mechanical properties in the upper region. Here average hardness is $3.8 \mathrm{GPa}$ and the distribution of $\mathrm{Ti}, \mathrm{V}$ and $\mathrm{Nb}$ is close to the equal-atomic one (Figure $6 \mathrm{c}$ ). These observations suggest that AITiVNb may be an interesting MPE system to develop further by SLM of elemental powder mixing.
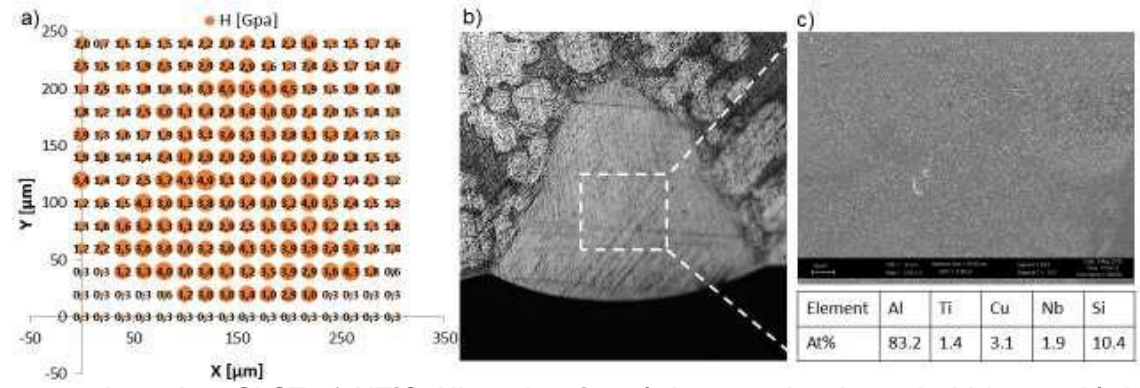

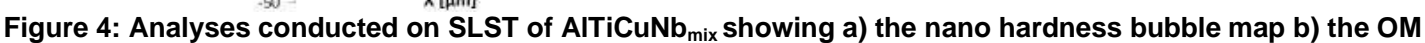
micrograph of the indented region and c) microstructure and composition of the dotted rectangular selection.
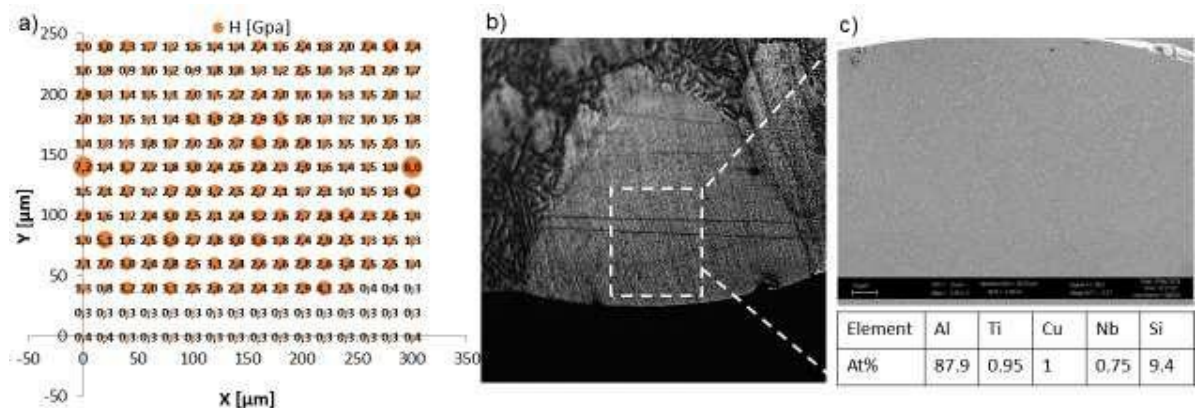

Figure 5: Analyses conducted on SLST of AITiCuNb mill showing a) the nano hardness bubble map b) the OM micrograph of the indented region and c) microstructure and composition of the dotted rectangular selection.
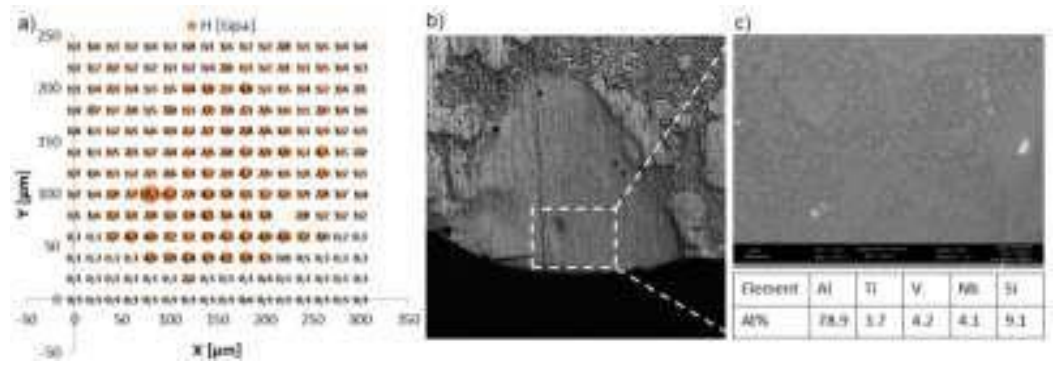

Figure 6: Analyses conducted on SLST of AITiVNb ${ }_{\text {mix }}$ showing a) the nano hardness bubble map b) the OM micrograph of the indented region and c) microstructure and composition of the dotted rectangular selection.

\section{Conclusions}

Methodologies for rapid exploration of new HEAs/MPEAs are important to develop new alloys by SLM. In the present work, two MPEAs, AITiCuNb and AITiVNb, likely to form a BCC single phase according to empirical solid solution rules, were studied. The cross sections of SLSTs obtained by SLM of equalatomic elemental powder mixtures of AITiCuNb

Image, mechanical and chemical analysis of SLSTs of MPEAs can be a useful tool to gain information about phase evolution of MPEAs during SLM. Our findings show that laser scan speed affects the remelting depth of the SLST of MPEAs. The AITiCuNbmill has the lowest remelting depths at any scanning speeds. Furthermore, the difference in chemical composition between AITiCuNb and AITiVNb affects the height of the SLST head. Then, we have also shown that nanoindentation testing, coupled with EDS analysis for chemical composition, could represent a useful tool to target promising MPE systems to be developed further by SLM. Particularly, we found that AITiCuNbmix has a low nano hardness and a strong tendency to $\mathrm{Cu}$ segregation, differently from AITiVNbmix which shows a significantly higher hardness and a close-to-equal-atomic distribution of elements. 


\section{Bibliography}

[1] J.W. Yeh, S.K. Chen, S.J. Lin, J.Y. Gan, T.S. Chin, T.T. Shun, C.H. Tsau, S.Y. Chang, Nanostructured high-entropy alloys with multiple principal elements: novel alloy design concepts and outcomes, Mater. Sci. Eng. 6 (5) (2004) 299-303.

[2] Y. Zhang, Y.J. Zhou, J.P. Lin, G.L. Chen, P.K. Liaw, Solid-solution phase formation rules for multicomponent alloys, Adv. Eng. Mater. 10 (6) (2008) 534-538.

[3] C. Li, J.C. Li, M. Zhao, Q. Jiang, Effect of aluminium contents on microstructure and properties of Al $x_{\mathrm{C}} \mathrm{CoCrFeNi}$, J. Alloys Compd. 504 S (2010) S515-S518.

[4] K.Y. Tsai, M.H. Tsai, J.W. Yeh, Sluggish diffusion in Co-Cr-Fe-Mn-Ni high entropy alloys, Acta. Mater. 61(13) (2013) 4887-4897.

[5] Y.J. Zhou, Y. Zhang, Y.L. Wang, G.L. Chen, Solid solution alloys of AlCoCrFeNiTix with excellent room-temperature mechanical properties, Appl. Phys. Lett. 90 (2007) 181904.

[6] Y. Qui, Y.J. Hu, A. Taylor, M.J. Styles, R.K.W. Marceau, A.V. Ceguerra, M.A. Gibson, Z.L. Liu, H.L. Fraser, N. Birbilis, A lightweight single-phase AITiVCr compositionally complex alloy, Acta. Mater. 123 (2017) 115-124.

[7] N.D. Stepanov, D.G. Shaysultanov, G.A. Salishchev, M.A. Tikhonovsky, Structure and mechanical properties of a lightweight AINbTiV high entropy alloy, Mater. Lett. 142 (2015) 153-155.

[8] O.N. Senkov, J.D. Miller, D.B. Miracle, C. Woodward, Accelerated exploration of multi-principal element alloys for structural applications, CALPHAD 50 (2015), 32-48.

[9] Y.F. Ye, Q. Wang, J. Lu, C.T. Liu, Y. Yang, High-entropy alloys: challenges and prospects, Mater. Today (2015).

[10] S. Guo, C.T. Liu, Phase stability in high entropy alloys: formation of solid-solution phase or amorphous phase, Progress in Natural Science: Materials International 21 (2011) 433-446.

[11]X. Yang, Y. Zhang, Prediction of high-entropy stabilized solid-solution in multi-component alloys, Mater. Chem. Phys. 132 (2012) 233-238.

[12] K.G. Pradeep, C.C. Tasan, M.J. Yao, Y. Deng, H. Springer, D. Raabe, Non-equiatomic high entropy alloys: Aprroach towards rapid alloy screening and property-oriented design, Mater. Sci. Eng. A 648 (2015) 183-192.

[13] D. Miracle, B. Majumdar, K. Wertz, S. Gorsse, New strategies and tests to accelerate discovery and development of multi-principal element structural alloys, Scr. Mater. 127 (2017) 195-200.

[14] D.B. Miracle, J.D. Miller, O.N. Senkov, C. Woodward, M.D. Uchic, J. Tiley, Exploration and development of high entropy alloys for structural applications, Entropy 16 (2014) 494-525.

[15] I. Gibson, D.W. Rosen, B. Stucker, 2015, Additive Manufacturing Technologies: 3D Printing, Rapid Prototyping, and Direct Digital Manufacturing, Springer, pp. 1-498.

[16] C. Haase, F. Tang, M.B. Wilms, A. Weisheit, B. Hallstedt, Combining thermodynamic modeling and 3D printing of elemental powder blends for high-throughput investigation of high-entropy alloys Towards rapid alloy screening and design, Mater. Sci. Eng. A 688 (2017) 180-189.

[17] B. Gwalani, A.V. Ayyagari, D. Choudhuri, T. Scharf, S. Mukherjee, M. Gibson, R. Banerjee Microstructure and wear resistance of an intermetallic-based Al0.25Ti0.75CoCrFeNi high entropy alloy.

[18] Mater. Chem. Phys. (2017)S. Guo, C. Ng, J. Lu, C.T. Liu, Effect of valence electron concentration on stability of fcc or bcc phase in high entropy alloys, J. Appl. Phys. 109 (2011) 103505.

[19] Y.Y. Zhao, T.G. NiehCorrelation between lattice distortion and friction stress in Ni-based equiatomic alloys Intermetallics, 86 (2017), pp. 45-50

[20] H. Song, F. Tian, Q.-M. Hu, L. Vitos, Y. Wang, J. Shen, N. Chen Local lattice distortion in highentropy alloys Phys. Rev. Mater., 1 (2017), Article 023404 